Mara Viveros 1

Fredy Gomez 1

Eduardo Otero ${ }^{1}$

\section{Las representaciones sociales sobre la esterilización masculina. El punto de vista de los orientadores del servicio de vasectomía en la Clínica del Hombre, en Bogotá, Colombia}

\author{
Social representations of male sterilization. \\ Vasectomy as viewed by professional counselors \\ at the Men's Clinic in Bogotá, Colombia
}

1 Programa Género, Mujer y Desarrollo, Centro de Estudios Sociales, Facultad de Ciencias Humanas, Universidad Nacional de Colombia. Carrera 50 no 27-70, Bloque B 5-6, Oficina 505, Bogotá, Colombia. Fax: (571) 3165137 mviveros@colomsat.net.co

\begin{abstract}
Vasectomy service providers at the Clinica del Hombre (Men's Clinic) in Bogotá, Colombia, construct their representations concerning male sterilization on the basis of biomedical discourse, their interaction with clinic users, and their own life experiences. During provision of such services, the counselors are the health professionals who have the closest contact with these clients of the clinic. Counselors are in charge of exploring their motivations in requesting the service, in addition to providing information allowing them to take a calm, sound decision. They also directly filter demand based on their experience, knowledge, and skills, in permanent negotiation between institutional criteria (number of children and gap between them, client's age, steadiness of couple's relationship, and conviction concerning the decision), and their perception and assessment of the client. An analysis of the counselors' representations of decision-making as pertaining to vasectomy indicates that it is a relatively simple and harmless procedure as well as a liberating option in terms of family planning.
\end{abstract}

Key words Vasectomy; Social Representations; Male Sterelization; Family Planning Services

Resumen Los oferentes del servicio de vasectomía en la Clínica del Hombre, en Bogotá, construyen sus representaciones sobre este método anticonceptivo con base en un discurso biomédico, en su interacción con el usuario y en sus propias experiencias de vida. En la oferta del servicio, los orientadores tienen el mayor contacto con los usuarios. Son los encargados de indagar los motivos para solicitar el servicio y dar información que permita tomar una decisión con tranquilidad y certeza. Adicionalmente, son quienes, de manera directa, filtran la demanda del servicio a partir de sus experiencias, conocimientos y destrezas. Esto, conforme a una permanente negociación que realizan entre los criterios institucionales (número y espaciamiento de los hijos, edad del cliente, estabilidad de la pareja y grado de seguridad en la decisión), y su percepción y valoración del usuario. Las representaciones de los orientadores estudiados sobre la toma de decisión de la vasectomía muestran que es presentada, por una parte, como un método fácil e inofensivo y, por otra, como una opción de planificación liberadora.

Palabras clave Vasectomía; Representaciones Sociales; Esterilización Masculina; Planeamiento Familiar 


\section{Introducción}

Con base en la idea prevaleciente de que la responsabilidad de la anticoncepción y de la reproducción es únicamente de las mujeres, todos los esfuerzos investigativos en esta área han estado orientados principalmente hacia ellas (Figueroa \& Liendro, 1994). La participación del varón en el proceso reproductivo aparece completamente diluida en la mayor parte de los análisis, razón por la cual vale la pena incluir la temática del varón como campo de estudio dentro de las investigaciones en salud reproductiva e interpretar su presencia en este proceso.

Desde esta perspectiva se está realizando una investigación sobre las imágenes y conceptos que se construyen socialmente en torno a uno de los métodos masculinos de anticoncepción, la esterilización quirúrgica (Viveros, 1995). Para tal objeto, se realizaron, de marzo a diciembre de 1996, quince entrevistas semiestructuradas a oferentes del servicio de vasectomía (médicos y orientadores), veinte a varones vasectomizados, cinco a algunas de sus esposas y a cinco grupos focales con hombres y mujeres usuarios de los programas de Profamilia (Asociación Pro-bienestar de la Familia), entidad privada de planificación familiar. En este artículo presentaremos algunos de los primeros resultados de esta investigación referidos únicamente a las representaciones de los orientadores del Programa de vasectomía de la Clínica del Hombre en Bogotá. La escasa cobertura que el Estado colombiano ha mantenido en este campo ha ocasionado que las acciones de Profamilia fueran, hasta hace pocos años, prácticamente las únicas que se registraran en el país. Con la apertura de sus "Clínicas para el Hombre”, en 1985 en Bogotá, Medellín y Cali, aumentó considerablemente el número de esterilizaciones masculinas realizadas. Así, de 10.312 esterilizaciones masculinas realizadas entre 1970 y 1984, se pasó a 44.618 en 1993, de las cuales el $40 \%$ se realiza en Bogotá. Llama la atención, sin embargo, la baja proporción de esterilizaciones masculinas (5\%), en relación al total de esterilizaciones de ambos sexos realizadas por Profamilia desde el inicio de su programa (Ordoñez \& Ojeda, 1994), sobre todo si se tiene en cuenta que la esterilización masculina es un método anticonceptivo tan eficaz como la esterilización femenina y técnicamente más simple de efectuar, sin necesidad de anestesia general y sin riesgos biológicos reconocidos (Population Reports, 1984).

Existen razones de diversa índole para las reticencias de los varones y de los proveedores de servicios de planificación familiar frente a este método. Sin embargo, las estrategias empleadas para aumentar su utilización han estado más orientadas a buscar resultados que a indagar sobre las raíces y causas de este comportamiento, ignorando la asignación diferencial de responsabilidades a mujeres y varones, en distintos ámbitos de sus vidas. Por otra parte, la interacción de los oferentes con los usuarios puede estar incidiendo en el bajo porcentaje de esterilizaciones masculinas. Al respecto, resulta pertinente preguntarse cómo es la relación que se establece entre los orientadores y los usuarios y cuáles son sus representaciones en relación con la vasectomía, el usuario y su proceso de toma de decisión.

\section{La relación del orientador con el usuario}

Para favorecer la relación orientador-usuario, la Clínica del Hombre ha intentado evitar las deficiencias más comunes en la prestación de los servicios en planificación familiar, buscando adecuar los horarios de atención a las necesidades de los usuarios, disminuyendo los trámites burocráticos que prolongan innecesariamente su tiempo de espera, preservando la privacidad de la orientación a través de la instalación de cubículos para atender a los usuarios y brindando al usuario la posibilidad de referir sus problemas y preocupaciones. Sin embargo, el carácter mismo de la orientación (para seleccionar un método anticonceptivo) no permite que el usuario exprese sus verdaderas necesidades y temores, ligados o no al método que va a escoger. La respuesta de los orientadores planteando que los temores expresados no tienen fundamento científico, a pesar de ser a veces correcta, no ayuda mucho a resolver las inquietudes personales del usuario, quien, a partir de ese momento se siente censurado para expresarse con libertad.

El orientador adopta con el usuario un tono pastoral y pedagógico. Por una parte guía y orienta al usuario hacia una "buena" decisión para él y, por la otra, enseña lo que se supone no sabe el paciente (aprovecha cada orientación para hacer "docencia”). Esto implica para el usuario ser remitido al lugar del que no tiene criterios suficientes para tomar la decisión anticonceptiva más adecuada para él (inmaduro) y del que no sabe (ignorante). Esta actitud de los orientadores puede relacionarse con el papel que han jugado los proveedores de servicios en la redefinición de la sexualidad y de la reproducción. Como lo plantea Tuirán (citado 
por Figueroa, 1996:297), "a través de lo que dicen (o no dicen) comunican la visión 'legítima' de las formas aceptables de conducir una vida sexual y procreativa. La relación entre el usuario y el profesional de la planificación familiar oculta una relación de poder en la cual los juicios y el conocimiento inherente a la práctica profesional se convierten en reglas establecidas que otorgan autoridad absoluta a los portadores del saber".

El nivel de relación del orientador con el usuario es muy superficial, si se tiene en cuenta el tipo de preguntas que se le formula, el tiempo que se le destina y el carácter unívoco de la comunicación, desarrollada alrededor de un guión predeterminado. Esta forma de estructurar la orientación respondería a las demandas implícitas o explícitas de la Institución de garantizar la eficacia y eficiencia del servicio (contribuyendo a alcanzar ciertas metas demográficas), y evitaría el riesgo de un excesivo involucramiento afectivo por parte del orientador.

Por otra parte, podría decirse que, por desconocimiento de las motivaciones reales de los usuarios, se posibilitan su idealización, la de su proceso de decisión y la de las circunstancias en las cuales se toma esta decisión. La relación orientador-usuario se enmarca dentro de un modelo funcionalista que le asigna al orientador los atributos de competencia técnica y neutralidad afectiva y al usuario los de motivación para el procedimiento, confianza en el personal que le brindan los servicios y acatamiento de sus competencias profesionales y humanas. Esto tiene por consecuencia que la percecpción que los orientadores tienen de los usuarios no corresponde a un conocimiento directo de ellos, sino al de las funciones que desempeñan en el marco de la orientación.

\section{Las representaciones de los orientadores del servicio de vasectomía}

Las representaciones de la esterilización masculina de los orientadores se construyen en el espacio institucional en el cual laboran y en contacto con las representaciones de los usuarios del programa. Uno de los elementos más importantes de las representaciones de los oferentes de este método anticonceptivo es el discurso biomédico que transmite información anatomo-fisiológica sobre el procedimiento quirúrgico y presenta al paciente como beneficiario del conocimiento médico. Esta información es interpretada y reelaborada por los propios oferentes del servicio a la luz de sus cono- cimientos, experiencias y actitudes en relación con la vida familiar, el comportamiento sexual, la historia reproductiva etc.

Para ilustrar la construcción de estas representaciones sobre la vasectomía, el usuario y la sexualidad, colocaremos en paralelo la descripción y el análisis de las respuestas de dos de los orientadores de la Clínica del Hombre (un hombre y una mujer).

\section{La vasectomía}

Los dos orientadores tienen en común la forma de presentar este método como una opción deseable e inocua para el usuario, ya que no ofrece complicaciones postquirúrgicas ni afecta negativamente ningún aspecto de su vida. Sin embargo, se diferencian en las ventajas que cada uno de ellos enfatiza. El orientador, no sólo asegura que es un método inofensivo sino que además mejora el posterior desempeño sexual del usuario. La orientadora sólo se refiere a que es un método que no ofrece complicaciones ("método fácil y más sencillo que una ligadura”). En uno y otro caso, la forma de realizar el ofrecimiento responde a la necesidad de hacer accesible el uso del método y de vencer los temores con los que llega el usuario.

En relación con los criterios de filtración de los usuarios, el orientador expresa que el principal parámetro es el número de hijos que tienen al momento de solicitar el servicio, mientras la orientadora adiciona el de la actitud de la pareja, traducida en sus niveles de estabilidad. En uno y otro caso, la vasectomía es colocada en relación con el tamaño ideal de la familia y con el modelo de pareja estable. En síntesis, los criterios de aprobación o rechazo del usuario potencial son fundamentalmente su alto grado de seguridad en la decisión y el buen nivel de estabilidad conyugal.

La orientadora plantea que el proceso de toma de decisión del usuario se realiza en pareja, de forma serena, con base en la información suministrada y por fuera del espacio institucional. En contraste, el orientador no hace ninguna referencia a la participación de la pareja ni al entorno que rodea dicha elección. Afirma que la decisión debe ser libre e informada y debe ser tomada por fuera del espacio y tiempo de la orientación. Estas respuestas no parecen considerar las múltiples eventualidades que una decisión de tal naturaleza implica en el ámbito familiar, conyugal y personal. Dicha determinación se percibe como un acto externo al de la orientación ya sea porque el usuario toma la decisión de antemano o porque la efectúa en un contexto no institucional. Es decir, aparece 
como un proceso que pertenece al ámbito privado del individuo y debe producirse idealmente en su "escenario natural", la familia.

Los orientadores destacan que ellos mismos, al haber vivido la experiencia de la vasectomía (él vasectomizado, ella esposa de un vasectomizado), garantizan la veracidad y seriedad de la información. La experiencia propia o cercana es uno de los ejes alrededor de los cuales los orientadores construyen su discurso y puede convertirse en el mejor y más convincente argumento para vencer los temores y desconfianzas de los usuarios frente al método.

\section{La vasectomía y la sexualidad}

Pese a que en Profamilia se están llevando a cabo acciones dirigidas a construir espacios de diálogo y reflexión en torno al concepto y significados de la sexualidad, se continúa hablando de ella únicamente desde el ámbito de la reproducción. "En lo de la sexualidad no nos metemos”, dicen unánimemente orientadores y médicos. El carácter de agencia de planificación familiar que tuvo la entidad hasta hace pocos años explica que la sexualidad continuara siendo un tema de discusión y educación ignorado al interior de la institución; sólo hace poco tiempo se consideró necesario trabajar sobre las concepciones de los prestatarios de servicios en torno a la sexualidad, buscando que los proveedores pudieran brindar a los usuarios información y consejería más pertinentes en materia sexual y reproductiva.

La orientadora, aunque reporta dedicar un tiempo especial en la orientación al tema de la sexualidad y diferenciarla de la reproducción, en la práctica sigue hablando de ella únicamente desde la perspectiva de sus efectos reproductivos. Por su parte, el orientador no reconoce explícitamente tal diferencia. La sexualidad aparece de manera implícita en las percepciones sobre la vasectomía, cuando la proponen como un método liberador, en tanto elimina el riesgo de los embarazos no deseados, permitiendo que la vida sexual resulte más placentera. El placer sexual y el mejor desempeño sexual del vasectomizado se convierten de esta manera en la promesa implícita en el discurso del orientador.

"Usted es el mismo, pero su vida sexual va a cambiar, va a ser más chévere, más placentera (...) su potencia sexual no la va a perder. Su vida sexual va a transcurrir igual a como está transcurriendo en este momento en que usted está sentado. Va a seguir eyaculando su semen, va a seguir teniendo erecciones, no va a cambiar en nada."
Por su parte la orientadora plantea que la vasectomía, como método definitivo, permite tener relaciones sexuales placenteras al brindar tranquilidad, eliminar el temor de embarazos no deseados y posibilitar el desarrollo positivo de la vida de pareja: "(La vasectomía es) una alternativa de planificación que puede permitirle a la persona tener relaciones sexuales en una forma placentera con su pareja, permitiéndose una tranquilidad de que no está haciendo embarazos que no está deseando (...) entonces yo creo que la vasectomía es una buena alternativa de planificación familiar cuando la persona ha decidido que no quiere tener más hijos (...) Aparte de ser un método de planificación, yo creo que puede ser un soporte para sentir tranquilidad dentro del desarrollo de su vida sexual, entonces eso puede llevar a tener un mejor desarrollo de su vida sexual y de pareja"

Es interesante comentar la diferencia entre los planteamientos de estos dos orientadores. Mientras el uno hace énfasis en la potencia sexual, la otra subraya el papel que puede jugar el hecho de separar la procreación de la sexualidad en una relación de pareja. Podrían atribuirse estos dos énfasis a diferencias de género según las cuales la sexualidad es percibida por los varones desde un ángulo de desempeño y por las mujeres desde una perspectiva más relacional.

\section{Los usuarios de los servicios de vasectomía}

El orientador se representa al usuario como una persona fundamentalmente temerosa: "entra ese usuario un poco temeroso y prevenido, a ver qué es lo que le van a hacer, qué es lo que le van a decir". El orientador explica esta actitud por la asociación que el usuario establece entre la vasectomía y el posible deterioro de su posterior desempeño sexual. Según el orientador, el usuario promedio teme perder, por la intervención quirúrgica, su potencia sexual, la capacidad de erección o el deseo sexual. Estas aprensiones son atribuidas a su supuesta ignorancia acerca de la utilización de este método, sin considerar las posibles situaciones de vida que pueden explicarlas. Además, se lo representa como miembro de una pareja estable, al interior de la cual se discute en forma serena el número de hijos. Sin embargo, al reportar las situaciones vividas por los usuarios, el orientador refiere circunstancias menos armoniosas como pueden ser las que rodean una decisión de aborto. Es decir, se observa un desfase entre la imagen construida del usuario y las situaciones reales atendidas por el oferente. 
El orientador caracteriza a los usuarios como hombres "machistas", que piensan que las decisiones son de su exclusiva competencia: "La expectativa de ellos - los usuarios - es que ser hombre es ser el que impone, aquí mando yo. Siempre se resalta ese machismo". Surge una oposición entre imagen y realidad: aunque las decisiones parecen tomarse de mutuo acuerdo al interior de la pareja, en realidad son asumidas o se desean asumir en forma individual, contradicción que no es percibida como tal por el orientador.

Los niveles de educación o información sexual de los usuarios son circunscritos por el oientador al conocimiento/información que tienen sobre los métodos de planificación familiar. Es decir, se homologan el estrato socioeconómico, el nivel educativo y el profesional y se relaciona con la información sobre los métodos anticonceptivos, el acceso a ellos, el nivel de participación en las decisiones reproductivas de la pareja y la posibilidad de tomar decisiones al respecto. Sus respuestas no diferencian el nivel educativo del grado de conocimiento sobre sexualidad y reproducción.

En relación con las asociaciones que los usuarios establecen, el orientador informa que muchos de ellos hacen la equivalencia entre vasectomía y castración. La aclaración ofrecida por el orientador se fundamenta en que “(...) la castración sólo se les hace a los animales, en particular al cerdo" y que la vasectomía, por el contrario, es un método escogido por hombres racionales, modernos y civilizados. A manera de hipótesis se puede plantear que una de las formas de oponerse a una asociación tan fuerte es asignarle a la vasectomía un significado positivo igualmente contundente, el de constituir una prueba de verdadera hombría.

Gran parte de los temores de los usuarios, reportada por los orientadores entrevistados, está relacionada con los recursos cognitivos de los que éstos disponen. En efecto, los orientadores perciben cierto desconocimiento de parte de los usuarios de la Clínica del Hombre de los aspectos relacionados con la anticoncepción y de la vasectomía propiamente dicha. Esta situación ha sido reforzada por las políticas de Profamilia que privilegiaron, durante largo tiempo como usuarias a las mujeres. Es decir, la tradición educativa de la institución estuvo volcada casi exclusivamente sobre la población femenina, creando una cultura anticonceptiva que excluyó a los varones de las decisiones reproductivas.

Es importante recalcar que una cultura anticonceptiva no sólo se crea con información "objetiva” sobre un procedimiento, sino a par- tir de un contexto relacional e intersubjetivo. Si bien muchos de los temores y expectativas con las que llegan los usuarios se relacionan con la carencia de información acerca de la vasectomía y de los servicios, tambien tienen que ver con los aspectos emocionales presentes en su toma de decisión. Es importante tener en cuenta que esta opción genera una serie de dudas que no pueden ser resueltas durante la orientación, por cuanto en ella se privilegia la perspectiva biomédica. Se ignora por ejemplo el hecho de que la vasectomía, por ser un método anticonceptivo definitivo, puede afectar la autopercepción del varón y las relaciones que éste establece con su entorno conyugal, familiar y social. Frente a las incertidumbres y angustias que esta situación genera, los orientadores no cuentan con recursos distintos a los que les puede ofrecer su destreza y experiencia profesional y personal.

Para ilustrar el desconocimiento de los aspectos subjetivos de la toma de decisión durante la orientación, podemos hacer referencia al criterio de selección de los usuarios privilegiado por los orientadores, el número de hijos. Este elemento, relacionado con factores como la edad y la estabilidad de la relación de pareja del usuario, constituye una condición suficiente para su aceptación en el servicio. Dicho parámetro aparece disociado de otros aspectos de la vida del usuario, tales como la importancia y el significado de la paternidad, el tamaño ideal de familia desde un punto de vista subjetivo, etc.

Al referirse a las motivaciones de los usuarios para practicarse la vasectomía, los orientadores subrayan sus temores ante la situación social y de violencia en el país, que vuelve incierto y poco promisorio el presente y el futuro de los hijos. Los describe de la siguiente manera: "Lo primero que le dicen a uno es que la sociedad está mala, está corrompida, ya no hay forma de vivir, (...) los hijos que tengo ya vieron cosas en un noticiero como que mataron, que acribillaron, que secuestraron, que violaron, lo ven en la prensa o en la misma comunidad (...), ven la sociedad tan descompuesta... Para qué traer más hijos si ya tengo los que quiero y los que tengo mire en lo que van".

La orientadora describe a los usuarios como personas que acuden al servicio en razón de "necesidades concretas y no por curiosidad". Añade que "aunque pueden ser machistas, tienen una valoración acerca de lo que es la mujer, la pareja y la familia".. Son descritos como personas muy seguras de sí mismas y que pocas veces se arrepienten de su decisión: "pueden ser machistas, eso no interesa (...), pero tienen 
un valor de lo que es la mujer, la pareja y la familia, saben lo que quieren y es raro ver a un hombre que venga y nos diga que se arrepiente de su decisión, eso se veía mucho en la clínica femenina (...)”. Esta descripción expresa una representación del usuario como alguien decidido por el sólo hecho de ser un varón - implícitamente define a las mujeres como seres indecisos -, como un ser racional, seguro, inteligente en sus actuaciones y decisiones y considerado con su entorno familiar y de pareja.

La orientadora clasifica a los usuarios en relación con su actitud frente a la vasectomía y los divide en dos grupos: los "negativos" y los "positivos”. El grupo de los "negativos”está conformado por aquellos que no se interesan por la información brindada durante la orientación: "casi siempre estos hombres se ponen fríos, empiezan a tocar el vidrio, me miran, miran para arriba, si traen a un niño se ponen a cuchiquearlo, miran el reloj... ¿Por qué? Porque ellos no quieren eso para ellos". Tambien hacen parte de él los hombres vasectomizados que demandan un reconocimiento social de su decisión como un acto de generosidad hacia su compañera. La orientadora explica esta actitud por "la excesiva importancia que otorgan los hombres a sus genitales". Los "positivos” son los usuarios que están interesados en recibir la información, a pesar de no estar seguros de tomar esta decisión, y aquellos que asumen la decisión de manera tranquila y sin reclamar ningún tipo de reconocimiento público. Este último subgrupo encarna, desde el punto de vista de la orientadora, el modelo del "hombre de verdad": seguro de sí mismo, solidario y discreto.

Las representaciones de los orientadores difieren en la forma de clasificar a los usuarios. El orientador los tipifica de acuerdo a su oficio, nivel educacional y socioeconómico, mientras que ella lo hace de acuerdo a su actitud frente a la decisión de esterilizarse. Pero tienen en común la idealización que ambos hacen del entorno que rodea su proceso de toma de decisión. Ninguno de ellos considera las dinámicas y relaciones de poder que se ponen en juego al interior de la pareja. Tampoco amplían el espectro de actores y ámbitos que pueden intervenir o incidir de manera importante en tal proceso (círculos de amigos, compañeros de trabajo, parientes, etc.). Finalmente, se puede afirmar que estas representaciones sobre el usuario y sobre el método desconocen y ocultan la dimensión de vida y las complejas circunstancias en las cuales la decisión se puede estar llevando a cabo. Método fácil-decisión segura y tranquila parecen ser los elementos que constituyen el núcleo figurativo alrededor del cual se construyen las representaciones de los oferentes sobre la vasectomía y el usuario.

\section{A manera de conclusión}

La relación entre orientadores y usuarios de los programas de vasectomía está enmarcada dentro de un modelo funcionalista que tiene por consecuencia una percepción de los usuarios a partir del rol que desempeñan en el marco de la orientación y de las representaciones de la vasectomía articuladas en torno al discurso biomédico. Por otra parte, las representaciones de los orientadores analizados sobre la toma de la decisión de la vasectomía están marcadas por su condición de género. El orientador tranquiliza al usuario haciendo referencias a los efectos positivos del método sobre su posterior desempeño sexual, valoración que como varón comparte con el usuario. En contraste, la orientadora resalta el mérito del hombre que toma esta decisión, la solidaridad masculina con la pareja y la conveniencia de esta elección para la familia, es decir, subraya los elementos relacionales de esta decisión. Los orientadores presentan la vasectomía como un método fácil e inofensivo y como una opción de planificación liberadora por cuanto elimina el temor al embarazo. Desde esta perspectiva, aparece como una opción que propicia las relaciones sexuales placenteras y el fortalecimiento de la relación de pareja. Se propone además como una elección de hombres racionales, modernos y civilizados que encarnarían un nuevo tipo de masculinidad. En este sentido se puede plantear que una de las formas más eficaces de oponerse a una asociación tan fuerte como la que se establece corrientemente entre vasectomía y castración es atribuirle a la vasectomía un significado positivo igualmente contundente, el de prueba y garantía de la verdadera hombría. 


\section{Referencias}

FIGUEROA, J. G. \& LIENDRO, E., 1994. Apuntes sobre la presencia del varón en la toma de decisiones reproductivas. In: Seminario sobre Masculinidad. México, DF: PUEG, Universidad Nacional Autónoma de México.

FIGUEROA, J. G., 1996. Comportamiento reproductivo y salud: reflexiones a partir de la prestación de servicios. In: Etica y Salud Reproductiva (C. G. Pérez, J. G. Figueroa \& M. C. Mejia, compiladores), pp. 289-317. México: PUEG/PUIS/Porrúa.

ORDOÑEZ, M. \& OJEDA, G., 1994. La Esterilización Femenina en Colombia. Bogotá: Encuestas Nacionales/PROFAMILIA.

POPULATION REPORTS, 1984. Esterilización masculina. Populations Reports, 4(Serie D).

VIVEROS, M., 1995. Representaciones y Prácticas Sociales de la Esterilización Masculina. Un Estudio de Caso en Bogotá. Proyecto de Investigación. Bogotá: Subprograma de Investigaciones del Programa de Género, Mujer y Desarrollo de la Facultad de Ciencias Humanas de la Universidad Nacional de Colombia. 